\title{
Analyse chromosomique des œufs humains non clivés après injection intracytoplasmique de spermatozoïde
}

Selva J., Bergère M., Frydman N., Rodrigues D., Fanchin R., Olivennes F., Frydman R. Laboratoire de Biologie de la Reproduction et Service de Gynécologie-Obstétrique, Hôpital A. Béclère, 157, Rue de la Porte de Trivaux, 92140 Clamart Cedex

\section{RESUME}

La microinjection intracytoplasmique d'un spermatozoïde dans l'ovocyte (ICSI), est une technique récente permettant d'obtenir des embryons pour des couples souffrant d'infertilité d'origine masculine sévère. Nous avons étudié les ovocytes non clivés 48 heures après l'injection. Cette analyse cytologique permet d'une part de déterminer le stade d'évolution du noyau spermatique dans le cytoplasme ovocytaire et donc de préciser l'étape de l'échec, d'autre part d'observer les chromosomes ovocytaires et leurs éventuelles anomalies. Après fécondation in vitro classique, dans $80 \%$ des cas l'échec est expliqué par une absence de fécondation mais environ $10 \%$ des absences de clivage sont dues à une condensation prématurée des chromosomes spermatiques. Notre étude a été menée sur deux périodes différentes. Pendant la première période, l'injection du spermatozoïde était faite dès que la membrane ovocytaire était rompue, pendant la deuxième période, l'aspiration était brièvement maintenue après rupture de la membrane, avant que le spermatozoïde soit injecté.

Les taux de fécondation ont été de $15 \%$ et de $54 \%$ respectivement au cours des deux périodes. L'analyse des ovocytes fixés pendant la première période a montré que dans $71 \%$ des cas il y avait absence complète de décondensation du noyau spermatique alors que cette situation ne représentait que $33 \%$ des cas de la deuxième série. Les anomalies de décondensation du noyau spermatique ont en revanche pu être observées plus fréquemment dans la deuxième série $(26,7 \%)$ que dans la première $(8,2 \%)$.

Il semble donc que la technique d'injection elle même puisse jouer un rôle dans l'évolution du spermatozoïde, et en ce sens l'analyse cytogénétique des oeufs constituerait un contrôle de qualité de la technique d'injection.

Dans les deux séries, la fréquence des ovocytes présentant une anomalie chromosomique était plus élevée qu'après une fécondation in vitro classique. Ceci devra être confirmé sur une plus grande série.

Mots clés : microinjection intra-cytoplasmique, ovocyte, spermatozoïde, chromosome.

\section{INTRODUCTION}

L'injection intra-cytoplasmique de spermatozoïde (ICSI) connaît un développement important depuis 2 ans. Elle devient la méthode de choix pour le traitement des infertilités masculines sévères dans de 
nombreux laboratoires $[6,16]$ mais ses résultats et en particulier son innocuité doivent encore être évalués. L'ICSI permet d'obtenir des fécondations dans le cas de caractéristiques spermatiques extrêmement défectueuses mais ceci pose la question du risque génétique que cela peut éventuellement entraîner. Jusqu'à présent, peu de déficits géniques ont été associés [4, 8] à des anomalies spermatiques.

L'analyse chromosomique des œufs non clivés après injection intra-cytoplasmique d'un spermatozoïde peut apporter plusieurs types d'informations : d'une part elle permet d'identifier le stade du blocage et donc parfois la cause de l'échec : œuf non fécondé sans aucun développement du noyau spermatique ou bien fécondation anormale, avec condensation prématurée des chromosomes spermatiques, en phase G1 par exemple (PCC).

Par ailleurs, l'analyse chromosomique des échecs de microinjection permet de préciser les anomalies chromosomiques ovocytaires après l'injection. Après fécondation in vitro classique de telles études ont permis de conclure à une incidence d'environ 20 à 30 $\%$ d'anomalies chromosomiques parmi les ovocytes non fécondés. De plus, si dans environ $80 \%$ des cas, l'absence de clivage est due à une absence totale de fécondation sans modification du noyau spermatique, par contre dans environ $10 \%$ des cas il y aurait fécondation, mais condensation prématurée des chromosomes spermatiques [9, 10]. Cette anomalie de la fécondation pourrait être le plus souvent liée à l'immaturité ovocytaire $[1,7]$. Elle serait plus rare après injection subzonale de spermatozoïde (SUZI) [11] qu'après une fécondation in vitro classique.

Ces résultats pourraient être différents après ICSI. En effet, ils peuvent être modifiés soit par la technique elle-même soit par les pathologies spermatiques ou de l'interaction ovocyte-spermatozoïde qui ont conduit à utiliser la méthode. Nous rappor- tons ici les résultats d'analyse chromosomique d'échecs d'ICSI effectuée au cours de 2 périodes : une première période de mise en place de la technique dans le laboratoire puis une seconde période où la technique a été modifiée et les résultats ont été améliorés.

Nos résultats suggèrent que l'analyse chromosomique des échecs d'ICSI pourrait être utilisée comme un contrôle de qualité de l'injection puisque les résultats paraissent différents au cours des 2 périodes.

\section{MATERIEL ET METHODES}

L'ICSI a été proposée pour 2 indications :

Oligoasthénospermie sévère avec moins de 300000 spermatozoïdes mobiles fléchants sélectionnés par migration dans un gradient de Percoll ( $80 \%$ des cas) ou bien échecs répétés de fécondation in vitro (au moins 3) avec des caractéristiques spermatiques modérément altérées. La première période comporte les injections effectuées entre le 1er Janvier 1994 et le 1er Avril 1994 dans le centre de Clamart (26 tentatives effectuées et analysées). Après une interruption, la deuxième période de microinjections analysées a été effectuée du 1er Novembre 1994 au 31 Décembre 1994 (19 tentatives analysées sur 40 effectuées).

Après une désensibilisation avec des agonistes de la Gonadolibérine ( $\mathrm{GnRH})$, les patientes ont été stimulées par de la gonadotrophine humaine ménopausique (HMG, HUMEGON, Organon, France).

Les ovocytes ont été ponctionnés 36 heures environ après injection d'hormone chorionique gonadotrope (HCG) en utilisant une sonde vaginale. Les traitements de sperme ont été conformes à la méthode décrite par A. Van Steirteghem et al. [16] : tout d'abord, un lavage en milieu de Earles additionné de $3 \%$ de sérum albumine bovine, une centrifugation à $1800 \mathrm{~g}$ pendant 5 minutes puis une migration dans un gra- 
dient de Percoll à 2 phases $(95 \%-47.5 \%)$ une centrifugation à $300 \mathrm{~g}$ pendant 20 minutes et une nouvelle centrifugation pendant 5 minutes à $1800 \mathrm{~g}$.

Les ovocytes ont été décoronisés par un traitement à la hyaluronidase $(80 \mathrm{UI} / \mathrm{ml})$ jusqu'à dissociation de la corona. Seuls les ovocytes présentant 1 globule polaire ont été injectés. Les ovocytes étaient maintenus par une pipette de contention (capillaire Clark étiré à un diamètre externe de 120 $\mu \mathrm{m}$ avec un diamètre interne de $30 \mu \mathrm{m}$ à l'aide d'étireuses Narishige ou Research Instruments, et d'une microforge Alcatel). Les pipettes d'injection ont été étirées à un diamètre externe de $8 \mu \mathrm{m}$, interne de $6 \mu \mathrm{m}$, coupées, rodées jusqu'à l'obtention d'un biseau de $45 \%$. Une pointe aiguë a été modelée à l'extrémité à l'aide de la forge.

L'injection a été pratiquée sur un microscope inversé Olympus IMT2 équipé de micromanipulateurs seringues et microinjecteurs (Narishige et Research instruments). Pendant la première période, l'injection du spermatozoïde était faite dès que la membrane ovocytaire était rompue ; pendant la deuxième période, l'aspiration était brièvement maintenue après rupture de la membrane avant que le spermatozoïde soit injecté. 97 ovocytes au cours de la première période puis 71 ovocytes au cours de la seconde période, non clivés et ne présentant pas de pronucleus 16 à 18 heures après l'ICSI ont été fixés dans une solution méthanol acide acétique 3:1 selon la méthode de Tarkowsi [15] après un choc hypotonique de 6 minutes dans une solution de citrate de sodium $1 \%$. Les lames ont été colorées au Giemsa.

\section{RESULTATS}

Au cours de la première série, le taux de fécondation (défini comme le nombre de zygotes à 2 pronoyaux 18-20 heures après injection rapporté au nombre total d'ovocytes matures injectés intacts après l'injec- tion) a été de $20.8 \%$, le taux de grossesse par cycle de ponction a été de $15 \%$ et le taux de grossesse par transfert de $21 \%$.

Au cours de la deuxième série, le taux de fécondation a été de $54 \%$, le taux de grossesse par cycle de ponction a été de $31 \%$ et le taux de grossesse par transfert de $33 \%$.

Les stades d'arrêt du développement après ICSI ont été différents au cours des deux périodes (Tableau 1).

Au cours de la première période (97 ovocytes), la grande majorité des ovocytes (71 $\%$ ne montraient pas du tout d'évolution du noyau spermatique tandis que les débuts de fécondation avec têtes décondensées $(6.1 \%)$ et les fécondations anormales avec condensation prématurée (PCC) des chromosomes spermatiques (2.1\%) étaient très rares.

Par contre, au cours de la seconde période (71 ovocytes) et malgré l'injection d'ovocytes matures, l'absence totale de décondensation ne représentait que $33.8 \%$ des cas alors que les arrêts au stade tête décondensée représentaient $12.6 \%$ des cas et les PCC $14.1 \%$ des cas. L'absence de spermatozoïde identifiable après fixation, événement très rare au cours de la première période $(2.1 \%$ des cas), a été beaucoup plus fréquent au cours de la seconde période $(25.3 \%$ des cas).

Les tableaux 2 et 3 décrivent l'aspect des chromosomes ovocytaires après échec de microinjection.

Les taux de succès techniques paraissent faibles puisque seulement $24 \%$ des ovocytes ont été caryotypables dans la première série et $30 \%$ dans la seconde série.

L'incidence des caryotypes normaux paraît faible $(33.3 \%$ pour la première période; $45.4 \%$ pour la seconde).

Les anomalies concernaient souvent des hyperploïdies (respectivement $45.8 \%$ et $22.7 \%$ pour les deux périodes soit globalement $33 \%$ ) sans qu'il soit en fait possible de 
Tableau 1 : Stade d'arrêt du développement après ICSI au cours des deux périodes.

\begin{tabular}{lcc}
\hline & $\begin{array}{c}\text { 1ère PERIODE } \\
(\mathbf{n = 9 7 )}\end{array}$ & $\begin{array}{c}\text { 2ème PERIODE } \\
(\mathbf{n}=\mathbf{7 1})\end{array}$ \\
\hline Aspect dégénéré & 18 & 10 \\
& $(18.5 \%)$ & $(14.1 \%)$ \\
Pas d'évolution du noyau du spermatozoïde & 69 & 24 \\
Spermatozoïde décondensé & $(71.1 \%)$ & $(33.8 \%)$ \\
& 6 & 9 \\
Condensation prématurée des chromosomes (PCC) & $(6.1 \%)$ & $(12.6 \%)$ \\
Pas de spermatozoïde & 2 & 10 \\
& $(2,1 \%)$ & $(\mathbf{1 4 . 1} \%)$ \\
\end{tabular}

Tableau 2 : Taux de succès du caryotype ovocytaire après échec de microinjection intracytoplasmique (ICSI).

\begin{tabular}{lcc}
\hline & $\begin{array}{c}\text { 1ère période } \\
(\mathbf{n = 9 7 )}\end{array}$ & $\begin{array}{c}\mathbf{2} \text { période } \\
(\mathbf{n}=\mathbf{7 1})\end{array}$ \\
\hline Ovocytes non analysables & 21 & 10 \\
Ovocytes en Métaphase II & 76 & 61 \\
Ovocytes en Métaphase II comptable & 24 & 22 \\
& $(24.7 \%)$ & $(30.9 \%)$ \\
\hline
\end{tabular}

distinguer les cassures chromosomiques des aneuploÏdies concernant les petits chromosomes en raison de la mauvaise qualité des chromosomes obtenus avec ce type de «matériel».

\section{DISCUSSION}

Le stade d'arrêt du développement dans la première série était habituellement très précoce. Le déroulement de la fécondation était soit complet jusqu'au premier clivage soit totalement absent mais, il n'y avait guère de blocage ou d'anomalie au cours de la formation des pronoyaux.
Ces résultats sont très similaires à ce que nous avions observé après injection subzonale (SUZI) [11] où $75 \%$ des œufs comportaient un spermatozoïde strictement intact, $7.8 \%$ des ovocytes présentaient un noyau décondensé et dans $2.2 \%$ des cas, la condensation des chromosomes était prématurée. Selon Flaherty et coll. [3] après ICSI, un spermatozoïde peut être injecté dans le cytoplasme ovocytaire et ressortir ou bien une nouvelle membrane cytoplasmique peut être reformée autour du noyau du spermatozoïde l'isolant ainsi du cytoplasme [5]. Certains des échecs de la première série pourraient être expliqués par la 
Tableau 3 : Anomalies chromosomiques parmi les ovocytes non fécondes après microinjection.

\begin{tabular}{lccc}
\hline & $\begin{array}{c}\text { lère Période } \\
(\mathbf{n = 9 7})\end{array}$ & $\begin{array}{c}\text { 2ème Période } \\
(\mathbf{n = 7 1})\end{array}$ & $\begin{array}{c}\text { Série complète } \\
(\mathbf{n = 1 6 8 )}\end{array}$ \\
\hline Ovocytes caryotypés & 24 & 22 & 46 \\
Caryotypes normaux & 8 & 10 & 18 \\
& $(33.3 \%)$ & $(45.4 \%)$ & $(39.1 \%)$ \\
Caryotypes hyperploïdes à plus & 11 & 5 & 16 \\
de 23 chromosomes ou fragments & $(45.8 \%)$ & $(22.7 \%)$ & $(34.7 \%)$ \\
Caryotypes hypoploïdes & 5 & & \\
& $(20.8 \%)$ & $(31.8 \%)$ & $(26 \%)$ \\
\hline
\end{tabular}

position subzonale ou extra-cytoplasmique du spermatozoïde. La technique cytogénétique casse les membranes et ne permet pas de préciser la position spatiale exacte du spermatozoïde donc de répondre à cette question.

Des observations effectuées en microscopie à fluorescence après coloration au Hœchst [2] ou bien en microscopie électronique [13] n'ont pas rapporté d'observation de spermatozoïde en position subzonale mais les effectifs étant très réduits, le risque d'extrusion secondaire du spermatozoïde lorsqu'il n'est pas injecté assez profondément doit être considéré. Les résultats de cette première période sont conformes aux observations de Soballa et coll. [12].

Au cours de la seconde période, nous avons modifié la technique en poussant le spermatozoïde plus loin dans l'ovocyte en aspirant davantage de cytoplasme. Ceci a permis d'améliorer le taux de fécondation, comme l'ont observé également d'autres auteurs [14], et a modifié les résultats des analyses d'échec.

Il semble donc que la technique elle-même modifie la fréquence des différents stades d'échec. En effet, au cours de la seconde période, l'incidence des blocages du déroule- ment de la fécondation paraît élevée. $14 \%$ de PCC et $12,6 \%$ d'arrêt au stade tête décondensée. Dans la deuxième série d'ICSI, l'incidence de ce type d'anomalie paraît même plus fréquente qu'après une fécondation in vitro classique malgré l'utilisation d'ovocytes matures qui est considérée comme la cause la plus fréquente de PCC. Ceci suggère l'existence de pathologies de l'interaction ovocyte-spermatozoïde soit liées à la technique, soit liées aux pathologies gamétiques (spermatiques ou ovocytaires) elles-mêmes. L'absence de spermatozoïde retrouvé dans $25 \%$ des ovocytes de la deuxième série est pour le moment inexpliquée. La technique d'injection s'étant améliorée il parait peu vraisemblable que le spermatozoïde n'ait pas été injecté. Il est possible que l'ADN du spermatozoïde ait subi une décondensation massive ne permettant plus son identification. Nous procédons actuellement à des colorations de ces ovocytes par un colorant spécifique (iodure de propidium) de l'ADN et qui permet de voir l'ADN même sous forme très décondensée et non identifiable à la coloration de Giemsa

La fréquence des anomalies chromosomiques ovocytaires était plus élevée dans notre série d'échecs d'ICSI (60.8\%) qu'après 
FIV classique (entre 20 et $30 \%$ ). La moitié des anomalies sont des excès du nombre de chromosomes (33\%). Pour les petits chromosomes nous n'avons pas pu distinguer les hyperploïdies vraies des cassures. L'ICSI pourrait donc induire des cassures chromosomiques plutôt que des aneuploïdies sur les oeufs non clivés.

Cependant, les éléments de surveillance des embryons se développant après ICSI sont très rassurants (caryotypes fotaux, examen à la naissance).

L'augmentation des caryotypes anormaux parmi les oeufs injectés non clivés est à confirmer sur un effectif plus large. Elle pourrait être à l'origine de l'absence d'évolution de l'œuf injecté et donc ne pas intervenir sur les embryons effectivement transférés.

\section{REFERENCES}

1. CALAFELL JM, BADENAS J, EGOZCUE J, SANTALO J. : Premature chromosome condensation as a sign of oocyte immaturity. Hum Reprod 1991 ; 6 : 1017-102

2. DUBEY AK, EMMI A, RHEE M, PENZIAS AS, LAYMAN, TURKSOY RN, DE CHERNEY AH, REINDOLLAR RH, DUCIBELLA T. : The status of human sperm following intracytoplasmic sperm injection (ICSI) in unfertilized oocytes. J. Ass. Repr. Gen. 1995, $12: 75$ S. Abstract OC-194. IXth World Congress on IVF, Vienna, Austria, April 3-7, 1995.

3. FLAHERTY SP, PAYNE D, SWANN NJ, MATTHEWS CD. : Etiology of failed and abnormal fertilization after intracytoplasmic injection. Hum Reprod $1994 ; 9$, sup.4, 38.

4. GASRMAN O., BURFEIND P., GUNTHER E., HAMEISTEEER H., SZPIRER C., HOYER-FENDER S. : sequence, expression and chromosomal assignement of a human sperm outer dense fiber gene. Mol. Reprod. Dev., 1993, 36, 407-418.

5. NG SC, LIOW SL, SATHANANTHAN H, BONGSO A, RATNAM SS. : Microinjection of human sperm directly into human oocytes. J of Assisted Reprod and Genet $1993 ; 10 ; 5: 337-352$.

6. PALERMO G., JORIS H., DEVROEY P., VAN STEIRTEGHEM. : Pregnancies after intracytoplasmic injection of a single spermatozoon into an oocyte. Lancet 1992 ; 340, 17-18.
7. PLACHOT M, CROZET N. : Fertilization abnormalities in human in vitro fertilization. Hum Reprod 1992 ; 7: suppl. 1, 89-94.

8. ROUSSEAUX-PREVOST R, LESUT P, COLLIER F, RIGOT JM, DALLA VENEZIA N, SAINT-POL P, DELAUNAY J, GAUTHIER A, ROUSSEAUX J. : Abnormal expression of protein 4.1 in spermatozoa of infertile men with teratospermia. Lancet $1994 ; 343: 764-5$.

9. SCHMIADY H, KENTENICH H. : Premature chromosome condensation after in vitro fertilization. Hum. Reprod $1989 ; 4,6: 689-695$

10. SELVA J, MARTIN-PONT B, HUGUES JN, RINCE P, FILLION C, HERVE F, TAMBOISE A. AND TAMBOISE E. «Cytogenetic study of human oocytes uncleaved after in vitro fertilization" Hum Reprod 1991, $6: 709-713$.

11. SELVA J, WOLF JP, RINCE P, RODRIGUES D, FRYDMAN R, JOUANNET P. : Cytogenetic analysis of human oocytes after subzonal insemination. Prenat Diagn 1993 ; 13 : 311-21.

12. SOBALLA M, SCHMIADY H, SCHMUTZLER AG, KREBS D. : Cytogenetics of failed microfertilization. Hum Reprod 1994 ; 9 : sup.4., 219

13. SOUSA M., TESARIK J. : Ultrastructural analysis of fertilization failure after intracytoplasmic sperm injection. Hum. Reprod. 1994, 9, 12, 2374-2380.

14. SVALANDER P, WIKLAND M, FORSBERG A.S, JAKOBSSON, BENGTSSON A.C. : Routine application of intracytoplasmic sperm injetcion (ICSI) for the treatment of severe male factor infertility. The American Fertility Society, program supplement. 1994, S 46 .

15. TARKOWSKI AK. : An air drying method for chromosome preparations of mouse eggs. Cytogenetics $1966 ; 3: 393-400$.

16. VAN STEIRTEGHEM A, NAGY $Z$, JORIS $H$, STAESSEN C, SMITZ J, WISANTO A. DEVROEY P. : High fertilization and implantation rates after intracytoplasmic sperm injection. Hum Reprod $1993 ; 8: 1061-66$.

\section{ABSTRACT}

\section{Cytologycal analysis of uncleaved oocytes after sperm injection}

The results of intracytoplasmic sperm injection still need to be assessed concerning both its efficiency and its possible risks for the children to be born. Cytogenetical analysis of unclea- 
ved oocytes after ICSI can give different types of information. It can help in determining the cause of the failure, checking the injection and specifying the development stage of the spermatozoa and its possible abnormalities. It also allows an evaluation of the possible chromosome abnormalities induced in the oocyte and subsequently of the safety of the procedure for the oocyte itself. After conventional in vitro fertilization (IVF) the main cause of the lack of cleavage is the total absence of fertilization but the premature condensation of the spermatozoa chromosomes (PCC) is observed in about $10 \%$ of the cases. This might be different after ICSI because of the procedure itself or because of the sperm defect which requires ICSI to achieve fertilization. We studied ICSI failures during two periods : the first one started at the beginning of the use of the technique in our laboratory and the second one followed, using a different technique (pushing the spermatozoa further in the oocyte by aspirating more vigourously oocyte cytoplasm). The fertilization rates were $15 \%$ and $54 \%$ in the two periods. In the first period the main cause of the failure was the total absence of evolution of the spermatozoa in the oocyte and it represented only $33 \%$ of the cases of the second period. In the second period the incidence of PCC increased and the total absence of evolution was less frequent while the incidence of chromosome fragmentation in the oocyte remained high. Our results suggest that the technique used for ICSI is very important to avoid the secondary extrusion of the spermatozoa. A possible increase of oocyte chromosome breakage has to be confirmed.

Key Words : intracystoplasmic sperm injection, oocyte, spermatozoa, chromosome. 